\title{
Dispersion relation for hadronic light-by-light scattering and the muon $g-2$
}

\author{
Gilberto Colangelo* \\ Albert Einstein Center for Fundamental Physics, Institute for Theoretical Physics, \\ University of Bern, Sidlerstrasse 5, 3012 Bern, Switzerland \\ E-mail: gilbertoditp.unibe.ch \\ Peter Stoffer* \\ Helmholtz-Institut für Strahlen- und Kernphysik (Theory) and \\ Bethe Center for Theoretical Physics, University of Bonn, 53115 Bonn, Germany \\ E-mail: stofferehiskp.uni-bonn.de
}

Martin Hoferichter

Institute for Nuclear Theory, University of Washington, Seattle, WA 98195-1550, USA

E-mail: mhofer@uw. edu

\section{Massimiliano Procura}

Fakultät für Physik, Universität Wien, Boltzmanngasse 5, 1090 Wien, Austria

CERN Theory Division, 1211 Geneva 23, Switzerland

E-mail: mprocura@univie.ac.at

\begin{abstract}
The largest uncertainties in the Standard Model calculation of the anomalous magnetic moment of the muon $(g-2)_{\mu}$ come from hadronic contributions. In particular, it can be expected that in a few years the subleading hadronic light-by-light (HLbL) contribution will dominate the theory uncertainty. We present a dispersive description of the HLbL tensor, which is based on unitarity, analyticity, crossing symmetry, and gauge invariance. Such a model-independent approach opens up an avenue towards a data-driven determination of the HLbL contribution to $(g-2) \mu$.

The dispersive approach defines unambiguously the pion-pole and the pion-box contribution to the HLbL tensor. Using Mandelstam's double-spectral representation, we have proven that the pion-box contribution coincides exactly with the one-loop scalar QED amplitude, multiplied by the appropriate pion vector form factors.
\end{abstract}

The 8th International Workshop on Chiral Dynamics, CD2015

29 June 2015 - 03 July 2015

Pisa, Italy

\footnotetext{
* Speaker.
} 


\section{Introduction}

The anomalous magnetic moment of the muon $(g-2)_{\mu}$ has been measured [1] and computed to very high precision, about $0.5 \mathrm{ppm}$ (see e.g. [2]). For more than a decade, a discrepancy has persisted between the experiment and the Standard Model prediction, now of about $3 \sigma$. Forthcoming experiments at FNAL [3] and J-PARC [4] aim at reducing the experimental error by a factor of 4 .

The main uncertainty of the theory prediction is due to strong interaction effects. At present, the largest error arises from hadronic vacuum polarisation which, however, is expected to be reduced significantly with the help of new data from $e^{+} e^{-}$experiments [2]. In a few years, the subleading ${ }^{1}$ hadronic light-by-light contribution will dominate the theory error. In present calculations of the HLbL contribution, systematic errors are difficult, if not impossible, to quantify. A new strategy is required to avoid the model dependence as far as possible, to provide a solid estimate of the theory uncertainties and to reduce them. In the recent past, lattice QCD has made remarkable progress in this direction, and may well become one of the main players in this field in the near future [7-11]. In [12,13], we have presented the first dispersive description of the HLbL tensor. By making use of the fundamental principles of unitarity, analyticity, crossing symmetry, and gauge invariance, we provide a model-independent approach that will allow a more data-driven determination of the HLbL contribution to $(g-2)_{\mu}$.

A different approach, which aims at a dispersive description of the muon vertex function instead of the HLbL tensor, has been presented in [14]. This latter approach makes a more ambitious use of dispersion relations, since it tries to represent the HLbL tensor dispersively and also to calculate the two-loop integral over the photon propagators via a dispersive representation. This means that one needs to solve the dispersion relation for the HLbL tensor (at fixed $q^{2}$ of the photons) and at the same time the one describing the dependence on the photon virtualities. In [14] it has been shown that such an approach reproduces the known result for the pion-pole contribution, but extending it to the full contribution seems like a formidable task, and it remains to be seen whether it can be carried out successfully.

Our approach is, in a sense, more modest because we calculate the two-loop integral over the photon propagators directly: this means that we only need to solve the dispersion relation for the HLbL tensor at fixed photon virtualities. If enough data on the photon $q^{2}$-dependence are available, this is sufficient. Even with this simplification, however, the difficulties to be overcome are quite nontrivial, as will be briefly illustrated below. Here, we report on the current status of our project and in particular on a recent improvement of our dispersive approach $[15,16]$. We have constructed a generating set of Lorentz structures that is free of kinematic singularities and zeros. This simplifies significantly the calculation of the HLbL contribution to $(g-2)_{\mu}$. Our dispersive formalism defines both the pion-pole and pion-box topologies in an unambiguous way. By constructing a Mandelstam representation for the scalar functions, we have been able to prove that the box topologies are equal to the scalar QED (SQED) contribution multiplied by pion vector form factors. Which means that at least for this specific contribution the photon $q^{2}$-dependence is completely under control.

\footnotetext{
${ }^{1}$ Even higher-order hadronic contributions have been considered in $[5,6]$.
} 


\section{Lorentz structure of the HLbL tensor}

In order to study the HLbL contribution to $(g-2)_{\mu}$, we need a suitable representation of the HLbL tensor, the hadronic Green's function of four electromagnetic currents, evaluated in pure QCD:

$$
\begin{aligned}
& \Pi^{\mu \nu \lambda \sigma}\left(q_{1}, q_{2}, q_{3}\right) \\
& \quad=-i \int d^{4} x d^{4} y d^{4} z e^{-i\left(q_{1} \cdot x+q_{2} \cdot y+q_{3} \cdot z\right)}\left\langle 0\left|T\left\{j_{\mathrm{em}}^{\mu}(x) j_{\mathrm{em}}^{v}(y) j_{\mathrm{em}}^{\lambda}(z) j_{\mathrm{em}}^{\sigma}(0)\right\}\right| 0\right\rangle .
\end{aligned}
$$

Gauge invariance requires the HLbL tensor to satisfy the Ward-Takahashi (WT) identities

$$
\left\{q_{1}^{\mu}, q_{2}^{v}, q_{3}^{\lambda}, q_{4}^{\sigma}\right\} \Pi_{\mu \nu \lambda \sigma}\left(q_{1}, q_{2}, q_{3}\right)=0
$$

where $q_{4}=q_{1}+q_{2}+q_{3}$. The HLbL tensor can be written a priori in terms of 138 basic Lorentz structures built out of the metric tensor and the four-momenta [17]. Our first task is to write the HLbL tensor in terms of Lorentz structures that satisfy the WT identities, while requiring at the same time that the scalar functions that multiply these structures be free of kinematic singularities and zeros. A recipe for the construction of these structures has been given by Bardeen, Tung [18], and Tarrach [19] for generic photon amplitudes. Gauge invariance imposes 95 linear relations between the 138 initial scalar functions. A generating set ${ }^{2}$ consisting of 43 elements can be constructed following Bardeen and Tung [18]. However, as it was shown by Tarrach [19], such a set is not free of kinematic singularities and has to be supplemented by additional structures. We find a redundant generating set of dimension 54:

$$
\Pi^{\mu \nu \lambda \sigma}\left(q_{1}, q_{2}, q_{3}\right)=\sum_{i=1}^{54} T_{i}^{\mu \nu \lambda \sigma} \Pi_{i}(s, t, u)
$$

where the scalar functions $\Pi_{i}$ are free of kinematic singularities and zeros. The Mandelstam variables are defined by $s=\left(q_{1}+q_{2}\right)^{2}, t=\left(q_{1}+q_{3}\right)^{2}, u=\left(q_{2}+q_{3}\right)^{2}$. There are only seven distinct

\footnotetext{
${ }^{2}$ In 4 space-time dimensions, there are two more linear relations, hence a basis consists of 41 elements [20, 21].
} 
Lorentz structures:

$$
\begin{aligned}
T_{1}^{\mu \nu \lambda \sigma}= & \varepsilon^{\mu \nu \alpha \beta} \varepsilon^{\lambda \sigma \gamma \delta} q_{1 \alpha} q_{2 \beta} q_{3 \gamma} q_{4 \delta} \\
T_{4}^{\mu \nu \lambda \sigma}= & \left(q_{2}^{\mu} q_{1}^{v}-q_{1} \cdot q_{2} g^{\mu \nu}\right)\left(q_{4}^{\lambda} q_{3}^{\sigma}-q_{3} \cdot q_{4} g^{\lambda \sigma}\right) \\
T_{7}^{\mu \nu \lambda \sigma}= & \left(q_{2}^{\mu} q_{1}^{v}-q_{1} \cdot q_{2} g^{\mu \nu}\right)\left(q_{1} \cdot q_{4}\left(q_{1}^{\lambda} q_{3}^{\sigma}-q_{1} \cdot q_{3} g^{\lambda \sigma}\right)+q_{4}^{\lambda} q_{1}^{\sigma} q_{1} \cdot q_{3}-q_{1}^{\lambda} q_{1}^{\sigma} q_{3} \cdot q_{4}\right) \\
T_{19}^{\mu \nu \lambda \sigma}= & \left(q_{2}^{\mu} q_{1}^{v}-q_{1} \cdot q_{2} g^{\mu \nu}\right)\left(q_{2} \cdot q_{4}\left(q_{1}^{\lambda} q_{3}^{\sigma}-q_{1} \cdot q_{3} g^{\lambda \sigma}\right)+q_{4}^{\lambda} q_{2}^{\sigma} q_{1} \cdot q_{3}-q_{1}^{\lambda} q_{2}^{\sigma} q_{3} \cdot q_{4}\right) \\
T_{31}^{\mu \nu \lambda \sigma}= & \left(q_{2}^{\mu} q_{1}^{v}-q_{1} \cdot q_{2} g^{\mu \nu}\right)\left(q_{2}^{\lambda} q_{1} \cdot q_{3}-q_{1}^{\lambda} q_{2} \cdot q_{3}\right)\left(q_{2}^{\sigma} q_{1} \cdot q_{4}-q_{1}^{\sigma} q_{2} \cdot q_{4}\right) \\
T_{37}^{\mu \nu \lambda \sigma}= & \left(q_{3}^{\mu} q_{1} \cdot q_{4}-q_{4}^{\mu} q_{1} \cdot q_{3}\right)\left(q_{3}^{v} q_{4}^{\lambda} q_{2}^{\sigma}-q_{4}^{v} q_{2}^{\lambda} q_{3}^{\sigma}+g^{\lambda \sigma}\left(q_{4}^{v} q_{2} \cdot q_{3}-q_{3}^{v} q_{2} \cdot q_{4}\right)\right. \\
& \left.\quad+g^{v \sigma}\left(q_{2}^{\lambda} q_{3} \cdot q_{4}-q_{4}^{\lambda} q_{2} \cdot q_{3}\right)+g^{\lambda v}\left(q_{3}^{\sigma} q_{2} \cdot q_{4}-q_{2}^{\sigma} q_{3} \cdot q_{4}\right)\right) \\
T_{49}^{\mu \nu \lambda \sigma \sigma}= & q_{3}^{\sigma}\left(q_{1} \cdot q_{3} q_{2} \cdot q_{4} q_{4}^{\mu} g^{\lambda \nu}-q_{2} \cdot q_{3} q_{1} \cdot q_{4} q_{4}^{v} g^{\lambda \mu}+q_{4}^{\mu} q_{4}^{v}\left(q_{1}^{\lambda} q_{2} \cdot q_{3}-q_{2}^{\lambda} q_{1} \cdot q_{3}\right)\right. \\
& \left.\quad+q_{1} \cdot q_{4} q_{3}^{\mu} q_{4}^{v} q_{2}^{\lambda}-q_{2} \cdot q_{4} q_{4}^{\mu} q_{3}^{v} q_{1}^{\lambda}+q_{1} \cdot q_{4} q_{2} \cdot q_{4}\left(q_{3}^{v} g^{\lambda \mu}-q_{3}^{\mu} g^{\lambda v}\right)\right) \\
- & q_{4}^{\lambda}\left(q_{1} \cdot q_{4} q_{2} \cdot q_{3} q_{3}^{\mu} g^{v \sigma}-q_{2} \cdot q_{4} q_{1} \cdot q_{3} q_{3}^{v} g^{\mu \sigma}+q_{3}^{\mu} q_{3}^{v}\left(q_{1}^{\sigma} q_{2} \cdot q_{4}-q_{2}^{\sigma} q_{1} \cdot q_{4}\right)\right. \\
& \left.\quad+q_{1} \cdot q_{3} q_{4}^{\mu} q_{3}^{v} q_{2}^{\sigma}-q_{2} \cdot q_{3} q_{3}^{\mu} q_{4}^{v} q_{1}^{\sigma}+q_{1} \cdot q_{3} q_{2} \cdot q_{3}\left(q_{4}^{v} g^{\mu \sigma}-q_{4}^{\mu} g^{v \sigma}\right)\right) \\
+ & q_{3} \cdot q_{4}\left(\left(q_{1}^{\lambda} q_{4}^{\mu}-q_{1} \cdot q_{4} g^{\lambda \mu}\right)\left(q_{3}^{v} q_{2}^{\sigma}-q_{2} \cdot q_{3} g^{v \sigma}\right)-\left(q_{2}^{\lambda} q_{4}^{v}-q_{2} \cdot q_{4} g^{\lambda v}\right)\left(q_{3}^{\mu} q_{1}^{\sigma}-q_{1} \cdot q_{3} g^{\mu \sigma}\right)\right) .
\end{aligned}
$$

All the remaining structures are just crossed versions of the above seven structures. As each structure fulfils the WT identities, both crossing symmetry and gauge invariance are implemented in a manifest way in the set $\left\{T_{i}^{\mu \nu \lambda \sigma}\right\}$. Since the scalar functions $\Pi_{i}$ are free of kinematic singularities, they are the well-suited quantities for a dispersive description.

\section{HLbL contribution to $(g-2)_{\mu}$}

The extraction of the HLbL contribution to $a_{\mu}=(g-2)_{\mu} / 2$ with the help of Dirac projector techniques is well-known [22]. With our decomposition of the HLbL tensor in 54 structures, this amounts to the calculation of the following two-loop integral:

$$
\begin{aligned}
a_{\mu}^{\mathrm{HLbL}}= & -\frac{e^{6}}{48 m_{\mu}} \int \frac{d^{4} q_{1}}{(2 \pi)^{4}} \frac{d^{4} q_{2}}{(2 \pi)^{4}} \frac{1}{q_{1}^{2} q_{2}^{2}\left(q_{1}+q_{2}\right)^{2}} \frac{1}{\left(p+q_{1}\right)^{2}-m_{\mu}^{2}} \frac{1}{\left(p-q_{2}\right)^{2}-m_{\mu}^{2}} \\
& \times \operatorname{Tr}\left(\left(\not p+m_{\mu}\right)\left[\gamma_{\rho}, \gamma_{\sigma}\right]\left(\not p+m_{\mu}\right) \gamma_{\mu}\left(\not p+q_{1}+m_{\mu}\right) \gamma_{\lambda}\left(\not p-q_{2}+m_{\mu}\right) \gamma_{v}\right) \\
& \times\left.\sum_{i=1}^{54}\left(\frac{\partial}{\partial q_{4} \rho} T_{i}^{\mu \nu \lambda \sigma}\left(q_{1}, q_{2}, q_{4}-q_{1}-q_{2}\right)\right)\right|_{q_{4}=0} \Pi_{i}\left(q_{1}, q_{2},-q_{1}-q_{2}\right) .
\end{aligned}
$$

After a Wick rotation of the momenta, five of the eight momentum integrals can be carried out with the technique of Gegenbauer polynomials [23]. We have checked that this Wick rotation is legitimate even in the presence of anomalous thresholds in the scalar functions $\Pi_{i}$. In analogy to the pion-pole contribution [24], a master formula for the full HLbL contribution to $(g-2)_{\mu}$ can be worked out:

$$
a_{\mu}^{\mathrm{HLbL}}=\frac{2 \alpha^{3}}{3 \pi^{2}} \int_{0}^{\infty} d Q_{1} \int_{0}^{\infty} d Q_{2} \int_{-1}^{1} d \tau \sqrt{1-\tau^{2}} Q_{1}^{3} Q_{2}^{3} \sum_{i=1}^{12} T_{i}\left(Q_{1}, Q_{2}, \tau\right) \bar{\Pi}_{i}\left(Q_{1}, Q_{2}, \tau\right),
$$



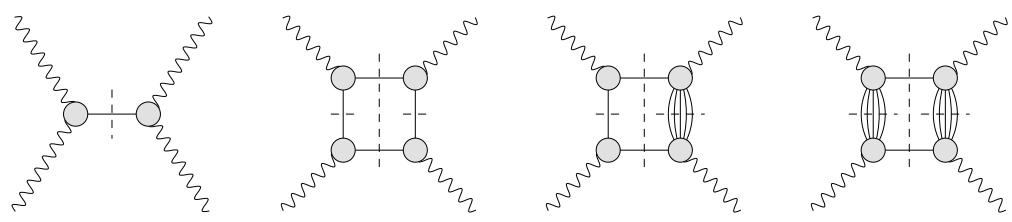

Figure 1: Unitarity diagrams according to the Mandelstam representation. Crossed diagrams are omitted.

where $\alpha=e^{2} /(4 \pi)$ and the $T_{i}$ are integration kernels. Only twelve independent linear combinations of the hadronic scalar functions $\Pi_{i}$ contribute, denoted by $\bar{\Pi}_{i}[16]$. They have to be evaluated for the reduced kinematics

$$
\begin{aligned}
s & =-Q_{3}^{2}, \quad t=-Q_{2}^{2}, \quad u=-Q_{1}^{2}, \\
q_{1}^{2} & =-Q_{1}^{2}, \quad q_{2}^{2}=-Q_{2}^{2}, \quad q_{3}^{2}=-Q_{3}^{2}=-Q_{1}^{2}-2 Q_{1} Q_{2} \tau-Q_{2}^{2}, \quad q_{4}^{2}=0 .
\end{aligned}
$$

\section{Mandelstam representation}

Although the scalar functions in the master formula (3.2) are needed only for the reduced kinematics (3.3), where the limit $q_{4} \rightarrow 0$ is taken, we define the dispersion relation in the Mandelstam variables of the four-point function with general kinematics and evaluate it only afterwards for the special case $q_{4} \rightarrow 0$. This procedure has the following advantage: the HLbL contribution to $(g-2)_{\mu}$ splits into contributions from different topologies, each of them linked to a specific subprocess, which is either data input or again a dispersively reconstructed quantity. These different contributions are discussed in the following.

Gauge invariance, encoded in the decomposition (2.3), leads to Lorentz structures $T_{i}^{\mu \nu \lambda \sigma}$ of mass dimension 4,6 , and 8 . Hence, we expect the scalar functions $\Pi_{i}$ to be rather strongly suppressed at high energies. This allows us to write down unsubtracted double-spectral (Mandelstam) representations for the $\Pi_{i}$ [25], i.e. parameter-free dispersion relations. The input to the dispersion relation are the residues at poles (due to single-particle intermediate states) and the discontinuities along branch cuts (due to two-particle intermediate states). Both are defined by the unitarity relation, in which the intermediate states are always on-shell. We neglect contributions from intermediate states consisting of more than two particles in the primary cut.

In the Mandelstam representation, the sum over intermediate states in the unitarity relations (for the primary and secondary cuts) translates into a splitting of the HLbL tensor into several topologies, shown in fig. 1. The first topology consists of the pion pole, i.e. the terms arising from a single pion intermediate state. This contribution is well-known [24] and given by

$$
\begin{aligned}
& \bar{\Pi}_{1}^{\pi^{0} \text {-pole }}=-\frac{\mathscr{F}^{0} \gamma^{*} \gamma^{*}}{\left(-Q_{1}^{2},-Q_{2}^{2}\right) \mathscr{F}^{0} \pi^{*} \gamma^{*}} \\
& Q_{3}^{2}+M_{\pi}^{2} \\
& \bar{\Pi}_{2}^{\pi^{0} \text {-pole }}=-\frac{\mathscr{F}_{\pi^{0} \gamma^{*} \gamma^{*}}\left(-Q_{1}^{2},-Q_{3}^{2}\right) \mathscr{F}_{\pi^{0} \gamma^{*} \gamma^{*}}\left(-Q_{2}^{2}, 0\right)}{Q_{2}^{2}+M_{\pi}^{2}},
\end{aligned}
$$

where $\mathscr{F}_{\pi^{0} \gamma^{*} \gamma^{*}}$ denotes the pion transition form factor (for off-shell photons but an on-shell pion).

The other topologies are obtained by selecting two-pion intermediate states in the primary cut. The sub-process $\gamma^{*} \gamma^{*} \rightarrow \pi \pi$ is again cut in the crossed channel. If we single out the pionpole contribution in both of the sub-processes, we obtain the box topologies for HLbL. For higher 
intermediate states in the crossed channel of $\gamma^{*} \gamma^{*} \rightarrow \pi \pi$, we obtain boxes with multi-particle cuts instead of poles in the sub-processes.

By explicitly constructing the Mandelstam representation, we have shown that the box topologies in the sense of unitarity have the same analytic structure as the one-loop sQED contribution, multiplied with pion electromagnetic form factors $F_{\pi}^{V}\left(q_{i}^{2}\right)$ for each of the off-shell photons (FsQED). The dispersion relation defines unambiguously this particular $q_{i}^{2}$ dependence. With the construction of the Mandelstam representation, we prove that FsQED and box topologies are the same. Note that the SQED loop contribution in terms of Feynman diagrams consists of boxes, triangles, and bulbs, but that the corresponding unitarity diagrams are just box topologies. This can be understood as follows: in SQED, the appearance of triangle and bulb diagrams is due to the seagull vertex, needed to ensure gauge invariance. In our formalism, gauge invariance is already encoded in the tensor decomposition (2.3). If the sQED contribution is projected on this tensor decomposition, which separates kinematics from dynamics, one can check that the dynamical singularities of the scalar functions $\Pi_{i}$ in sQED are the ones of a box topology.

The equivalence of the pion-box topologies with FsQED allows us to derive compact expressions for the contribution to the scalar functions $\Pi_{i}$ in terms of two-dimensional Feynman parameter integrals. In the limit $q_{4} \rightarrow 0$, they are given by

$$
\Pi_{i}^{\pi-\operatorname{box}}\left(q_{1}^{2}, q_{2}^{2}, q_{3}^{2}\right)=F_{\pi}^{V}\left(q_{1}^{2}\right) F_{\pi}^{V}\left(q_{2}^{2}\right) F_{\pi}^{V}\left(q_{3}^{2}\right) \frac{1}{16 \pi^{2}} \int_{0}^{1} d x \int_{0}^{1-x} d y I_{i}(x, y)
$$

where

$$
\begin{aligned}
& I_{1}(x, y)=-\frac{2}{3} \frac{(1-2 y)(1-2 x-2 y)(1-6 x(1-x))}{\Delta_{123}^{2}}, \\
& I_{4}(x, y)=-\frac{2}{3} \frac{(1-2 x)(1+2 x(1-3 x(1-2 y)-6 y(1-y)))}{\Delta_{123}^{2}}, \\
& I_{7}(x, y)=-\frac{4}{3} \frac{(1-2 x)^{2}(1-2 y)^{2} y(1-y)}{\Delta_{123}^{3}}, \\
& I_{16}(x, y)=\frac{4}{3} \frac{x(1-2 x) y(1-2 y)}{\Delta_{213} \Delta_{13}}\left(\frac{1}{\Delta_{213}}+\frac{1}{\Delta_{13}}\right), \\
& I_{19}(x, y)=-\frac{4}{3} \frac{(1-2 x)^{2}(1-2 y) y(1-y)(1-2 x-2 y)}{\Delta_{213}^{3}}, \\
& I_{31}(x, y)=-\frac{8}{3} \frac{x^{2}(1-x)(1-2 x)^{3} y(1-2 y)}{\Delta_{213} \Delta_{13}}\left(\frac{1}{\Delta_{213}^{2}}+\frac{1}{\Delta_{213} \Delta_{13}}+\frac{1}{\Delta_{13}^{2}}\right) \\
& I_{39}(x, y)=\frac{4}{3} \frac{(1-2 x)(1-2 y)^{2} y(1-y)(1-2 x-2 y)}{\Delta_{123}^{3}}, \\
& I_{42}(x, y)=-\frac{4}{3} \frac{x(1-2 x) y(1-2 y)(1-6 y(1-y))}{\Delta_{321} \Delta_{21}}\left(\frac{1}{\Delta_{321}}+\frac{1}{\Delta_{21}}\right) \\
& I_{50}(x, y)=0
\end{aligned}
$$

and

$$
\begin{aligned}
\Delta_{i j k} & =M_{\pi}^{2}-x y q_{i}^{2}-x(1-x-y) q_{j}^{2}-y(1-x-y) q_{k}^{2}, \\
\Delta_{i j} & =M_{\pi}^{2}-x(1-x) q_{i}^{2}-y(1-y) q_{j}^{2} .
\end{aligned}
$$


The remaining functions $\Pi_{i}$ that enter the master formula can be obtained with crossing relations permuting only $q_{1}, q_{2}$, and $q_{3}$, which are still valid in the limit $q_{4} \rightarrow 0$.
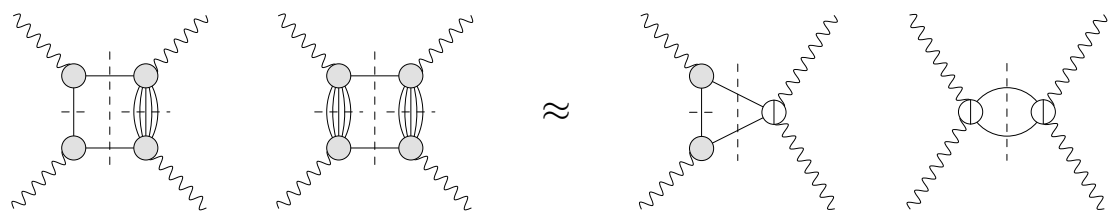

Figure 2: Partial-wave approximation of multi-particle intermediate states in the secondary cut.

We treat the contribution from topologies with higher intermediate states in a partial-wave picture. This means that the multi-particle cut is approximated by a polynomial, as illustrated in fig. 2. The dispersive formulation allows us to describe here the effect of two-pion rescattering in the primary channel. In [12], we already discussed the $S$-wave contribution. The new Lorentz decomposition allows us to include also higher partial waves. The contribution of these topologies is given by dispersion integrals over products of $\gamma^{*} \gamma^{(*)} \rightarrow \pi \pi$ helicity partial waves. The Born terms of the sub-process have to be properly subtracted to avoid double-counting with the box topologies.

\section{Conclusion and outlook}

Using the Mandelstam representation for the hadronic scalar functions $\Pi_{i}$, we have split $a_{\mu}^{\mathrm{HLbL}}$ into three contributions: pion-pole contributions, box topologies, and $\pi \pi$-rescattering contributions:

$$
a_{\mu}^{\mathrm{HLbL}}=a_{\mu}^{\pi^{0} \text {-pole }}+a_{\mu}^{\mathrm{box}}+a_{\mu}^{\pi \pi}+\ldots
$$

where the dots denote neglected higher intermediate states in the primary cut ${ }^{3}$. The input quantities in this dispersive description are the pion transition form factor $\mathscr{F}_{\pi^{0} \gamma^{*} \gamma^{*}}\left(q_{i}^{2}, q_{j}^{2}\right)$, the pion electromagnetic form factor $F_{\pi}^{V}\left(q_{i}^{2}\right)$, and the $\gamma^{*} \gamma^{*} \rightarrow \pi \pi$ helicity partial waves. In the absence of experimental data on the doubly-virtual processes, these quantities will be reconstructed again dispersively [12, 26-33].

We stress that the dispersive formalism defines unambiguously both the pion-pole and pionbox contribution. They are treated without any approximation. For the two-pion rescattering contribution a partial-wave expansion is employed.

So far we have limited the discussion to pions although the formalism can be extended without substantial changes to higher pseudoscalar poles $\left(\eta, \eta^{\prime}\right)$ or $K K$ intermediate states [34-37].

The dispersive approach presented here provides a first model-independent description of HLbL scattering and shows a path towards a more data-driven evaluation of the HLbL contribution to $(g-2)_{\mu}$. A careful numerical study is currently under way to identify the experimental input with the largest impact on the theory uncertainty.

\footnotetext{
${ }^{3}$ Marc Knecht suggested to give a name to the ellipsis, since at some point it will have to be discussed and estimated. In the future, it might therefore be convenient to call the ellipsis $a_{\mu}^{\text {residual }}$ or something similar.
} 


\section{Acknowledgments}

The speakers (GC and PS) thank the conference committee for the excellent organisation of the Chiral Dynamics 2015 conference. We thank J. Bijnens, J. Gasser, M. Knecht, B. Kubis, H. Leutwyler, A. Nyffeler, and S. Scherer for useful discussions. Financial support by the Swiss National Science Foundation, the DFG (CRC 16, "Subnuclear Structure of Matter"), and the DOE (Grant No. DE-FG02- 00ER41132) is gratefully acknowledged.

\section{References}

[1] G. Bennett et al. (Muon $(g-2)$ Collaboration), Phys. Rev. D73, 072003 (2006), [arXiv:hep-ex/0602035].

[2] T. Blum et al., (2013), arXiv:1311.2198 [hep-ph].

[3] J. Grange et al. (Muon $g$-2), (2015), arXiv:1501.06858 [physics.ins-det].

[4] N. Saito (J-PARC $g$-2/EDM), AIP Conf. Proc. 1467, 45 (2012).

[5] A. Kurz, T. Liu, P. Marquard and M. Steinhauser, Phys. Lett. B734, 144 (2014), [arXiv:1403.6400 [hep-ph]].

[6] G. Colangelo, M. Hoferichter, A. Nyffeler, M. Passera and P. Stoffer, Phys. Lett. B735, 90 (2014), [arXiv:1403.7512 [hep-ph]].

[7] M. Hayakawa, T. Blum, T. Izubuchi and N. Yamada, PoS LAT2005, 353 (2006), [arXiv:hep-lat/0509016].

[8] T. Blum, M. Hayakawa and T. Izubuchi, PoS LATTICE2012, 022 (2012), [arXiv:1301.2607 [hep-lat]].

[9] T. Blum, S. Chowdhury, M. Hayakawa and T. Izubuchi, Phys. Rev. Lett. 114, 012001 (2015), [arXiv:1407.2923 [hep-lat]].

[10] J. Green, O. Gryniuk, G. von Hippel, H. B. Meyer and V. Pascalutsa, (2015), arXiv:1507.01577 [hep-lat].

[11] T. Blum et al., Phys. Rev. D93, 014503 (2016), 1510.07100.

[12] G. Colangelo, M. Hoferichter, M. Procura and P. Stoffer, JHEP 1409, 091 (2014), [arXiv:1402.7081 [hep-ph]].

[13] G. Colangelo, M. Hoferichter, B. Kubis, M. Procura and P. Stoffer, Phys. Lett. B738, 6 (2014), [arXiv:1408.2517 [hep-ph]].

[14] V. Pauk and M. Vanderhaeghen, Phys. Rev. D90, 113012 (2014), [arXiv:1409.0819 [hep-ph]].

[15] P. Stoffer, Dispersive Treatments of $K_{\ell 4}$ Decays and Hadronic Light-by-Light Scattering, $\mathrm{PhD}$ thesis, University of Bern, 2014, arXiv:1412.5171 [hep-ph].

[16] G. Colangelo, M. Hoferichter, M. Procura and P. Stoffer, JHEP 1509, 074 (2015), [arXiv:1506.01386 [hep-ph]].

[17] R. Leo, A. Minguzzi and G. Soliani, Nuovo Cim. A30, 270 (1975).

[18] W. A. Bardeen and W. Tung, Phys. Rev. 173, 1423 (1968), [Erratum-ibid. D4, 3229 (1971)].

[19] R. Tarrach, Nuovo Cim. A28, 409 (1975). 
[20] G. Eichmann, C. S. Fischer, W. Heupel and R. Williams, (2014), arXiv:1411.7876 [hep-ph].

[21] G. Eichmann, C. S. Fischer and W. Heupel, Phys. Rev. D92, 056006 (2015), 1505.06336.

[22] J. Aldins, T. Kinoshita, S. J. Brodsky and A. Dufner, Phys. Rev. D1, 2378 (1970).

[23] J. L. Rosner, Annals Phys. 44, 11 (1967).

[24] M. Knecht and A. Nyffeler, Phys. Rev. D65, 073034 (2002), [arXiv:hep-ph/0111058].

[25] S. Mandelstam, Phys. Rev. 112, 1344 (1958).

[26] F. Niecknig, B. Kubis and S. P. Schneider, Eur. Phys. J. C72, 2014 (2012), [arXiv:1203.2501 [hep-ph]].

[27] S. P. Schneider, B. Kubis and F. Niecknig, Phys. Rev. D86, 054013 (2012), [arXiv:1206.3098 [hep-ph]].

[28] M. Hoferichter, G. Colangelo, M. Procura and P. Stoffer, Int. J. Mod. Phys. Conf. Ser. 35, 1460400 (2014), [arXiv:1309.6877 [hep-ph]].

[29] M. Hoferichter, B. Kubis, S. Leupold, F. Niecknig and S. P. Schneider, Eur. Phys. J. C74, 3180 (2014), [arXiv:1410.4691 [hep-ph]].

[30] R. García-Martín and B. Moussallam, Eur. Phys. J. C70, 155 (2010), [arXiv:1006.5373 [hep-ph]].

[31] M. Hoferichter, D. R. Phillips and C. Schat, Eur. Phys. J. C71, 1743 (2011), [arXiv:1106.4147 [hep-ph]].

[32] B. Moussallam, Eur. Phys. J. C73, 2539 (2013), [arXiv:1305.3143 [hep-ph]].

[33] M. Hoferichter, B. Kubis and D. Sakkas, Phys. Rev. D86, 116009 (2012), [arXiv:1210.6793 [hep-ph]].

[34] F. Stollenwerk, C. Hanhart, A. Kupść, U.-G. Meißner and A. Wirzba, Phys. Lett. B707, 184 (2012), [arXiv:1108.2419 [nucl-th]].

[35] C. Hanhart, A. Kupść, U.-G. Meißner, F. Stollenwerk and A. Wirzba, Eur. Phys. J. C73, 2668 (2013), [arXiv:1307.5654 [hep-ph]].

[36] B. Kubis and J. Plenter, Eur. Phys. J. C75, 283 (2015), [arXiv:1504.02588 [hep-ph]].

[37] C. W. Xiao et al., (2015), arXiv:1509.02194 [hep-ph]. 